sions in the incident and prevalent networks many YBGBM are embedded in, proper messaging is needed to account for the negative perceptions that surround it. Without this messaging, continued underuse in this important population will expand rather reduce HIV disparities.

Sources of Support: JHU Center for AIDS Research NIAID P30AI094189 CDC Category C Demonstration Project PS12-1201 NIH Diversity Supplement 3R01DA031030-04S1.

12.

\section{MEASURING ANTIRETROVIRAL ADHERENCE AMONG YOUNG PEOPLE LIVING WITH HIV: OBSERVATIONS FROM A REAL-TIME MONITORING DEVICE VERSUS SELF REPORT}

Nicholas Tarantino, $P h D^{1}$, Laura Whiteley, $M D^{2}$, Lacey Craker, $M P H^{2}$, Trisha L. Arnold, $\mathrm{MS}^{3}$, Leandro A. Mena, MD, MPH ${ }^{4}$,

Larry K. Brown, $M D^{2}$

${ }^{1}$ Alpert Medical School of Brown University; ${ }^{2}$ Rhode Island Hospital; ${ }^{3}$ Jackson State University; ${ }^{4}$ University of Mississippi Medical Center.

Purpose: Consistent adherence to antiretroviral therapy (ART) among young people living with HIV (YPLH) is a public health priority. Electronic monitoring devices such as Wisepill are important tools for capturing real-time adherence via mobile internet access. Understanding the limitations of these devices, however, is also critical. The current study is drawn from an adherence intervention trial for YPLH from the United States. We compare self-report (SR) adherence data to data collected via Wisepill, and examine associations between ARTadherence levels and young people's virologic functioning.

Methods: Sixty-six YPLH were recruited from an HIV clinic to participate in a trial of Battle Viro, a smartphone gaming app designed to improve ART adherence. Participants were assessed for outcomes over 14 weeks at four time points: T1 and four (T2), eight (T3), and 14 (T4) weeks after T1. Adherence was measured by Wisepill and SR by calculating the percent of antiretroviral doses taken in the past seven days. Descriptive analyses were used to compare SR to Wisepill data. In addition, correlations and a linear regression were conducted to explore factors hypothesized to explain SR-Wisepill discrepancies.

Results: Participants were aged 16 to 26 (mean age $=22$ ) and identified primarily as African American (97\%) and male (77\%). Rates of SR adherence for T1 to T4 were $90 \%, 92 \%, 90 \%, 87 \%$ while rates of Wisepill adherence were $64 \%, 59 \%, 45 \%$, and $34 \%$. The correlation between SR-Wisepill measurements ranged from .19 to .41. A decreasing rate of Wisepill adherence occurred even as rates of viral suppression increased from $6 \%$ (T1) to $41 \%$ (T4). SR and Wisepill adherence at each time point had similar correlations with viral load at T4 (rs $=-.24$ to -.56). Two YPLH characteristics emerged as independent predictors of a mean SR-Wisepill discrepancy score: newly diagnosed YPLH had lower discrepancy scores than other YPLH ( $B=$ $-.22,95 \% \mathrm{CI}=.42, .02, \mathrm{p}=.032)$ and frequency of recent marijuana use was positively associated with discrepancy score $(\mathrm{B}=.06,95 \% \mathrm{CI}=.00$, $.13, \mathrm{p}=.048$ ).

Conclusions: Given SR adherence levels and the increasing rate of viral suppression, ART intake based on Wisepill observations was likely an underestimation. Nevertheless, Wisepill adherence paralleled SR adherence in its association with young people's viral load at the end of 14 weeks. This suggests that the device should still be considered when assessing for predictors of HIV-related health functioning. Further research is needed to uncover which YPLH are at risk for overreporting adherence behaviors as well as not consistently using electronic monitoring devices including perhaps ART-experienced patients and youth endorsing heavy substance use.

Sources of Support: Research reported in this abstract was supported by the National Institute of Child Health and Human Development and the National Institute of Mental Health of the National Institutes of Health under award numbers R01HD074846 and T32MH078788.

\section{PLATFORM RESEARCH PRESENTATION III: GLOBAL ADOLESCENT HEALTH EQUITY}

13.

\section{FROM TOBACCO-RELATED PRODUCTS TO SMOKING: RESULTS FROM A LONGITUDINAL STUDY}

Christina Akre, $P h D^{1}$, Yara Barrense-Dias, $M A^{1}$, André Berchtold, $P h D^{2}$, Joan-Carles Suris, $M D, M P H, P h D^{1}$

${ }^{1}$ Lausanne University Hospital; ${ }^{2}$ Lausanne University.

Purpose: To assess longitudinal factors related to smoking among youths depending on smoking status at baseline.

Methods: Data were drawn from the two first waves (T0 in 2016 and T1 in 2017) of the GenerationFRee longitudinal study conducted in eleven post-mandatory schools in the Canton of Fribourg, Switzerland. Participants ( $\mathrm{N}=1606$, mean age 16.2 at baseline [15-24], 45.8\% females) filled out a web-based self-administered anonymous questionnaire which aimed at assessing their lifestyle. Participants were asked at $\mathrm{T} 1$ whether they smoked (Group 1, N=571) or not (Group $2, \mathrm{~N}=1035$ ). Groups were compared on the use of tobacco-related products (TRP) (e-cigarettes, hookah, snus, and smokeless tobacco) during the past 12 months, use of other substances (alcohol, cannabis) and socio-demographic (age, gender, family structure, socioeconomic status, academic track [student/apprentice]) variables at T0 separately by smoking status at T0. Groups were compared at the bivariate level and afterwards at the multivariate level using a logistic regression analysis to assess the variables associated with being a smoker at T1 using non-smokers as the reference category. Data are presented as odds ratios (OR) with 95\% confidence interval. Results: Smokers at T0. At the bivariate level, smokers at T1 were significantly more likely to be apprentices and showed a clear trend ( $\mathrm{p}=.052)$ to be less likely to live in an intact family. At the multivariate level, both variables remained significant: they were more likely to be apprentices (OR: 2.32 [1.28:4.22]) and to live in a non-intact family (OR: 1.75 [1.01:3.04]). Non-smokers at T0. At the bivariate level, those who became smokers at T1 were significantly more likely to have used TRPs ( $45.7 \%$ vs. $18.2 \%$; $<<.001$ ), to have misused alcohol, and to have used cannabis at T0. In the multivariate analysis, having used TRPs (OR: 3.05 [1.92:4.85]) and cannabis (OR: 2.89 [1.41:5.90]) remained significant. Moreover, when the analysis were repeated separately by product, they remained significant (E-cigarettes: 2.46 [1.54:3.91]; Smokeless tobacco: 1.88 [1.12:3.15]; Snus: 2.05 [1.07:3.92]; Hookah: 2.68 [1.73:4.14]).

Conclusions: These longitudinal results show an important association between using TRPs and starting to smoke and seem to confirm the gateway effect of products such as hookah, snus, smokeless tobacco, and electronic cigarettes. Therefore the potential harmful impact of these substances should not be underestimated. The association with cannabis is also an important result given that in Switzerland cannabis is mostly consumed in joints including tobacco. As an important percentage of non-smoking youths try different TRPs and they seem to be an entry point to cigarette consumption, TRPs 
should be part of substance use screening among adolescents. Similarly, tobacco prevention programs should be broadened to include tobacco-related products.

Sources of Support: The Programme Intercantonal de Lutte contre la Dépendance au Jeu (PILDJ) and the Canton of Fribourg.

14.

\section{TRAPPED: YOUNG WOMEN IN URBAN UTTAR PRADESH REFLECT ON THEIR MOBILITY \\ Luciana Estelle Hebert, $P h D^{1}$, Suchi Bansal, MPH ${ }^{1}$, Shirley Yan, BA ${ }^{1}$, Marquez Rhyne ${ }^{1}$, Harshita Seth, MA ${ }^{2}$, Sanjay Shukla, MASW ${ }^{2}$, Alicia Menendez, PhD, MA ${ }^{1}$, Melissa L. Gilliam, MD, $M P H^{1}$ \\ ${ }^{1}$ University of Chicago; ${ }^{2}$ Operation Asha.}

Purpose: Mobility is a key indicator of empowerment for girls and young women and has significant implications for their educational, economic, social, and health status, including their ability to access and use sexual and reproductive health services. This mixed methods study used novel methodologies to describe how gender affects mobility among youth in urban Uttar Pradesh, India.

Methods: Body mapping sessions, in which life narratives are illustrated on a full-size silhouette of the narrator's body, were conducted with 30 young women and 10 young men ages 14-17 years to explore contextual factors related to gender. Story circle sessions, in which participants share personal stories following a narrative prompt, were conducted with 43 young women and 31 young men ages $15-24$ years to explore the role of gender in education, home and community, and expectations for the future. Surveys were completed with 275 young men and women aged 15-24 years to measure limitations on mobility both within and outside the community.

Results: Each of the three methodologic approaches revealed restrictions and limitations on young women's mobility that were not experienced by young men. Through body mapping workshops, young women reported not being allowed to be outside the house unless they were chaperoned or on their way to school and parental warnings against talking to anyone in their community. In story circle sessions, young women shared stories of prohibitions placed on their movement by parents or older brothers. Experiences of street harassment were common, and used as justification for limiting girls' movement, keeping them at home, or requiring chaperones. Cumulatively, these restrictions left girls and young women feeling isolated. Restrictions on movement were also cited by young women as barriers to going to school and working outside of the home. Only $36 \%$ of young women reported they were able to go to the market alone, compared to $93 \%$ of young men; $62 \%$ of young women reported they could only go to the market with someone, and $3 \%$ said not at all. While $76 \%$ of males reported they could travel to a health facility alone, only $15 \%$ of female youth were allowed to do so; $83 \%$ said only if accompanied. While $97 \%$ of young men said they were allowed to go to places outside their community alone, only $30 \%$ of young women were allowed, with $64 \%$ allowed to do so only when accompanied. All differences in mobility were statistically significant $(p<.001)$.

Conclusions: Compared to young men, young women in Lucknow face many restrictions on their mobility, which influences their access to education, health services, and labor opportunities. Harassment, traditional gender norms, and family dynamics reinforce these mobility limitations. Programs and policies seeking to improve the sexual and reproductive health of women in India must consider the daily context that shapes the lives of women and girls. Barriers to mobil- ity remain a key challenge to empowering young women and girls in India.

Sources of Support: Funding for this project was provided by the Bill and Melinda Gates Foundation.

15.

\section{FACTORS ASSOCIATED WITH EMOTIONAL DISTRESS AND SUICIDALITY AMONG INTERNATIONAL COLLEGE STUDENTS}

Lindsay A. Taliaferro, $P h D, M P H^{1}$, Jennifer J. Muehlenkamp, $P h D^{2}$, Sathya B. Jeevanba, $B A^{3}$

${ }^{1}$ University of Central Florida; ${ }^{2}$ University of Wisconsin-Eau Claire; ${ }^{3}$ University of Missouri-Kansas City.

Purpose: Suicide ranks as the second leading cause of death for college students. Although enrollment of international students in American colleges has increased, researchers have failed to examine suicide risk among this unique population. The current study aimed to address gaps in the literature regarding the factors associated with emotional distress and suicidality among a sample of international college students.

Methods: The analytic sample include 334 international students of typical college age (i.e., 18 to 26) from two Midwestern and two Southeastern universities. Most students identified as female (56.0\%) and Asian (62.0\%), and 93.7\% were in the U.S. on an F1 academic Visa. Participants completed an anonymous online survey administered between 2013 and 2017. Emotional distress was measured with the Depression, Anxiety, and Stress Scales short form, and suicidality was assessed with the Suicide Behaviors Questionnaire. Participants also completed measures that assessed potential risk factors (perceptions of entrapment, cultural stress, family conflict, perfectionism, interpersonal needs, ethnic discrimination) and protective factors (cultural sanctions against suicide, ethnic identity, and social support). Pearson correlation tests were performed to examine bivariate relationships between each risk and protective factor and the mental health outcomes (alpha $=.05$ ). Significant variables were subsequently entered together, with gender and age, into linear regression analyses to determine the factors most strongly related to greater emotional distress and suicidality during the preceding year.

Results: In bivariate tests, factors significantly associated with greater emotional distress included higher levels of entrapment $(\mathrm{r}=.24)$, cultural stress $(\mathrm{r}=.18)$, family conflict $(\mathrm{r}=.16)$, unmet interpersonal needs $(\mathrm{r}=.53)$, and ethnic discrimination $(\mathrm{r}=.16)$. Similarly, factors significantly associated with greater suicidality were higher levels of entrapment $(\mathrm{r}=.30)$, cultural stress $(\mathrm{r}=.29)$, family conflict $(\mathrm{r}=.24)$, perfectionism $(\mathrm{r}=.17)$, unmet interpersonal needs $(\mathrm{r}=.35)$, and ethnic discrimination $(r=.24)$, and lower levels of social support $(r=-.15)$. When all significant factors were examined together in a regression analysis $F(7,259)=55.71, p<.001$, higher levels of entrapment $(\beta=.33$, $\mathrm{t}=11.00, \mathrm{p}<.001)$, unmet interpersonal needs $(\beta=.07, \mathrm{t}=2.54, \mathrm{p}<.05)$, and ethnic discrimination $(\beta=.11, t=2.50, p<.05)$ were significantly associated with increased emotional distress. Only unmet interpersonal needs $(\beta=.11, \mathrm{t}=2.60, \mathrm{p}=.01)$ remained significantly associated with greater past-year suicidality in a multivariate regression analysis $\mathrm{F}(9,249)=6.16, \mathrm{p}<.001$.

Conclusions: Findings provide initial evidence regarding factors associated with suicide risk among international college students. Clinicians working with international students and prevention programmers targeting this population should address students' perceptions of entrapment, ethnic discrimination, and especially unmet interpersonal needs in efforts to decrease/prevent students' feel- 